\title{
RAS Mutational Status in Advanced Colorectal Adenocarcinoma Treated With Anti-angiogenics: Preliminary Experience With Liquid Biopsy
}

\author{
BELÉN GARCÍA DE SANTIAGO ${ }^{1}$, MIRIAM LÓPEZ-GÓMEZ ${ }^{2}$, PEDRO DAVID DELGADO-LÓPEZ ${ }^{3}$, \\ ANA JIMÉNEZ GORDO ${ }^{2}$, FERNANDO NERIA ${ }^{4}$, ISRAEL JOHN THUISSARD-VASALLO ${ }^{4}$, \\ CÉSAR GÓMEZ-RAPOSO ${ }^{2}$, FRANCISCO ZAMBRANA TEVAR ${ }^{2}$, JUAN MORENO-RUBIO ${ }^{5}$, \\ ALICIA MARTÍNEZ HERNÁNDEZ ${ }^{1}$, IRENE IGLESIAS ${ }^{6}$ and ENRIQUE CASADO ${ }^{2}$ \\ ${ }^{1}$ Department of Pharmacy, Infanta Sofía University Hospital, FIIB HUIS HHEN, Madrid, Spain; \\ ${ }^{2}$ Department of Oncology, Infanta Sofía University Hospital, FIIB HUIS HHEN, Madrid, Spain; \\ ${ }^{3}$ Department of Neurosurgery, Burgos University Hospital, Burgos, Spain; \\ ${ }^{4}$ Faculty of Biomedical Science and Health, Universidad Europea, Madrid, Spain; \\ ${ }^{5}$ Clinical Oncology Group, IMDEA Food Institute, CEI UAM+CSIC, Madrid, Spain; \\ ${ }^{6}$ Department of Pharmacology, Pharmacognosy and Botany, \\ Faculty of Pharmacy, Complutense University Madrid, Madrid, Spain
}

\begin{abstract}
Aim: To determinate molecular changes in the downstream epidermal growth factor receptor signaling pathway using serial liquid biopsies in patients with metastatic colorectal tumors ( $m C R C)$ under anti-angiogenic treatment. Patients and Methods: Determination of RAS mutation in primary tissue samples from colorectal tumors was performed in the 23 patients included in the study at diagnosis using quantitative-polymerase chain reaction. Sequential mutations were studied in circulating tumor (ct) DNA obtained from plasma samples. Results: Twenty-three patients with RAS-mutated primary tumors were included. In the first ctDNA determination, 17 of these patients were found to have wild-type RAS status. Remarkably, three out of these 17 wild-type cases changed to RAS-mutated in subsequent ctDNA assays. Conclusion: Serial liquid biopsies in patients with $\mathrm{mCRC}$ might be a useful tool for identifying changes in the RAS mutation status in patients who had undergone previous anti-angiogenic therapy. The
\end{abstract}

This article is freely accessible online.

Correspondence to: Belén García De Santiago, PharmG, Department of Pharmacy, Infanta Sofía University Hospital, FIIB HUIS HHEN, Paseo de Europa 34, San Sebastián de los Reyes, 28702 Madrid, Spain. Tel: +34 911915231, Fax: +34 911914017, e-mail: belengsantiago@yahoo.es

Key Words: Colorectal cancer, RAS mutations, circulating tumor DNA, anti-angiogenic therapies, liquid biopsy. understanding of these changes might help to better define the landscape of $m C R C$ and be the path to future randomized studies.

Colorectal cancer (CRC) is an aggressive disease associated with a poor outcome in a subset of patients. Approximately $22 \%$ of CRCs are metastatic (mCRC) at initial diagnosis and about $70 \%$ will develop metastatic relapse (1). With the arrival of directed therapies against epidermal growth factor receptor (EGFR) signaling pathway for patients without mutations in KRAS proto-oncogene, GTPase (KRAS), NRAS proto-oncogene, GTPase $(N R A S)$ and B-RAF protooncogene, serine/threonine kinase $(B R A F)$ genes, overall survival has increased. The use of anti-angiogenic based chemotherapy has also revolutionized mCRC treatment. However, the impact of anti-angiogenics on the molecular profile of patients with $R A S$-mutated tumors is unclear. It has been hypothesized that $K R A S$-mutated tumors treated with vascular-directed therapy might change to a wild-type $K R A S$ status (2). Drug-induced hypoxia has been suggested as a possible explanation for this, and has been related to overexpression of wild-type KRAS (3). However, to date, only a few studies have examined in depth the consequences of these changes in the evolution of the disease.

Detection of mutation status of RAS genes in circulating tumor DNA (ctDNA) from blood samples seems to be a simple and non-invasive alternative to testing of primary tumors (4). It can easily be done at different time points in the course of the disease, providing information on dynamic changes occurring in the genotype of mCRC cells. 
Our hypothesis is that performing serial liquid biopsies in patients with mCRC under anti-angiogenic treatment might be a useful tool for establishing whether clonal selection occurs in patients with CRC under anti-angiogenic treatment. One of our objectives was to determine whether such changes remain over time or disappear as vascular endothelial growth factor (VEGF) inhibition is withdrawn.

\section{Patients and Methods}

We present a retrospective cohort study performed at the Infanta Sofía University Hospital in Madrid, Spain, from June 2018 to June 2019. The study was approved by the Local Ethics Committee (reference number: PI-4292).

Patients included in the study had mCRC with any mutation in RAS/RAF/mitogen-activated protein kinase: 17 patients showed mutation in KRAS oncoprotein and six patients in NRAS oncoprotein. All patients had received anti-VEGF treatment within the previous 6 months or were under such therapy at the time of the study. We performed assays of ctDNA for every patient included in the study, at the time of study inclusion and subsequently every 2 months, and finally at the time of radiological progression. A computed tomographic scan was performed every 2 months to exclude tumor progression. The Response Evaluation Criteria in Solid Tumors (RECIST) were used to establish progression of the disease (5).

Paraffin-embedded primary tumor specimens containing at least $70 \%$ tumor cells were selected for each patient. A polymerase-chain reaction (PCR) was performed to amplify 139 bp of exon 2, exon 3 and 4 in KRAS using specific primers. PCR primer sequences/conditions are available upon request. Further mutations in NRAS/BRAF were tested when no mutation in KRAS exons was found. When no NRAS mutation was found, BRAF V600E mutation was analyzed. The efficiency and quality of the polymerase-chain reaction (PCR) amplification were confirmed by running the products on a $2 \%$ agarose gel. A negative control containing all the components of the PCR except the template was included in each PCR reaction. DNA-amplified products were purified using a QuickStep $^{\mathrm{TM}} 2$ 96-Well PCR Purification Kit (Edge BioSystems, Gaithersburg, MD, USA), according to the manufacturer's instructions. Amplification products were bidirectionally sequenced via the fluorescence dye terminator method in a multi-capillary DNA. The presence of mutation was accepted when its chromatographic peak height was $25 \%$ or higher thas the peak of the wild-type reference.

For liquid biopsy, two analytic kits were used: Idylla ${ }^{\mathrm{TM}} \operatorname{ctKRAS}$ Mutation Assay, which globally covered 21 mutations: seven mutations in exon 2 (codon 12 and 13), nine mutations in exon 3 (codon 59 and 61) and five mutations in exon 4 (codon 117 and 146); and Idylla ${ }^{\mathrm{TM}}$ ctNRAS/BRAF Mutation Assay, which covered 23 mutations: eight NRAS mutations in exon 2 (codon 12 and 13), six NRAS mutations in exon 3 (codon 59 and 61), four NRAS mutationn in exon 4 (codon 117 and 146), and five BRAF mutations in exon 15 (codon 600, V600E mutation). The sensitivity (weighted average) and the sensitivity range (most prevalent $R A S$ mutations) of Idylla ${ }^{\mathrm{TM}}$ ctRAS was $0.4 \%(0.2-0.5 \%)$ for $K R A S, 1.5 \%(0.5-1.3 \%)$ for NRAS and $0.4 \%(0.4 \%)$ for $B R A F$.

Data were analyzed by two independent statistics experts from the European University in Madrid using IBM SPSS Statistics 23 (IBM, Armonk, NY, USA).
Table I. Baseline demographic data of patients included in the study $(n=23)$.

\begin{tabular}{|c|c|c|}
\hline Characteristic & & Value \\
\hline \multirow[t]{2}{*}{ Gender, n (\%) } & Female & $8(34.8 \%)$ \\
\hline & Male & $15(65.2 \%)$ \\
\hline Age, years & Mean \pm SD & $67.3 \pm 9.8$ \\
\hline \multirow{3}{*}{$\begin{array}{l}\text { Location of primary } \\
\text { tumor, } \mathrm{n}(\%)\end{array}$} & Rectum & $9(39.1 \%)$ \\
\hline & Colon & $13(56.5 \%)$ \\
\hline & Colorectum & $1(4.4 \%)$ \\
\hline \multirow{3}{*}{$\begin{array}{l}\text { Primary characteristics, } \\
\mathrm{n}(\%)\end{array}$} & Lymph node metastases & $11(47.8 \%)$ \\
\hline & $\begin{array}{l}\text { Perivascular/ } \\
\text { lymphatic invasion }\end{array}$ & $8(34.8 \%)$ \\
\hline & Microsatellite instability & $1(4.4 \%)$ \\
\hline \multirow[t]{3}{*}{ Stage at diagnosis* } & II & $1(4.3 \%)$ \\
\hline & III & $4(17.4 \%)$ \\
\hline & IV & $18(78.3 \%)$ \\
\hline \multirow[t]{3}{*}{ CEA } & Median (IQR) & $2.5(1.3-86.6)$ \\
\hline & $<5 \mu \mathrm{g} / 1$ & $14(60.9 \%)$ \\
\hline & $\geq 5 \mu \mathrm{g} / 1$ & $9(39.1 \%)$ \\
\hline \multirow[t]{3}{*}{ LDH } & Median (IQR) & $230(173-272)$ \\
\hline & $<250 \mathrm{U} / 1$ & $14(60.9 \%)$ \\
\hline & $\geq 250 \mathrm{U} / 1$ & $9(39.1 \%)$ \\
\hline \multirow[t]{4}{*}{ CA 19.9} & Median (IQR) & $23(9-107.5)$ \\
\hline & $<40 \mathrm{U} / \mathrm{ml}$ & $13(56.5 \%)$ \\
\hline & $>40 \mathrm{U} / \mathrm{ml}$ & $9(39.1 \%)$ \\
\hline & Unknown & $1(4.4 \%)$ \\
\hline \multirow{2}{*}{$\begin{array}{l}\text { Liver metastasis, } \\
\mathrm{n}(\%)\end{array}$} & Synchronous & $14(60.9 \%)$ \\
\hline & Metachronous & $2(8.7 \%)$ \\
\hline \multirow{4}{*}{$\begin{array}{l}\text { Treatment received, } \\
\mathrm{n}(\%)\end{array}$} & Aflibercept & $1(4.3 \%)$ \\
\hline & Regorafenib & $0(0.0 \%)$ \\
\hline & Bevacizumab & $21(91.3 \%)$ \\
\hline & Bevacizumab+aflibercept & t $1(4.3 \%)$ \\
\hline \multirow{5}{*}{$\begin{array}{l}\text { Number of chemotherapy } \\
\text { lines prior to } L B, n(\%)\end{array}$} & 0 & $12(52.2 \%)$ \\
\hline & 1 & $7(30.4 \%)$ \\
\hline & 2 & $3(13.0 \%)$ \\
\hline & 3 & $0(0.0 \%)$ \\
\hline & 4 & $1(4.3 \%)$ \\
\hline \multirow{2}{*}{$\begin{array}{l}\text { Primary tumor mutation, } \\
\mathrm{n}(\%)\end{array}$} & KRAS & $17(73.9 \%)$ \\
\hline & NRAS & $6(26.1 \%)$ \\
\hline
\end{tabular}

CA 19.9: Cancer antigen 19.9; CEA: carcinoembryonic antigen; IQR: interquartile range; LB: liquid biopsy; LDH: lactate dehydrogenase; SD: standard deviation. *American Joint Committee on Cancer $8^{\text {th }}$ edition.

\section{Results}

Twenty-three patients were included. Table I describes the demographic data of the study cohort. The mean age at diagnosis was $67.3 \pm 9.8$ years. Only one patient exhibited microsatellite instability, with loss of expression of $\mathrm{MSH} 2$. Prior to liquid biopsy, 21 patients had been treated with bevacizumab, one with aflibercept, and one had received both drugs. The median time from the initiation of antiangiogenics to the performance of the first liquid biopsy was 4 (range=1-28) months. Among primary tumors, 17 and 6 carried KRAS and NRAS mutations, respectively. No patient harbored $B R A F$ mutation. The first ctDNA analysis showed 
that the RAS mutation status had changed to wild-type in 17 patients $(73.9 \%)$, whereas six patients had maintained their RAS oncogene mutation. Subsequent ctDNA analysis identified three additional $R A S$ mutations among these 17 (17.6\%) patients (KRAS exon 2, KRAS G13D in two patients and $K R A S G 12 R$ in one patient). Interestingly, the $R A S$ mutation status of these three patients returned to their initial RAS mutation status after a median duration of treatment with anti-angiogenic drugs of 7 (range=6-9) months. Our study was not able to determine the precise moment when the RAS mutational status changed, as sequential biopsies were performed on a two-month basis.

\section{Discussion}

Identification of ctDNA by liquid biopsy in mCRC tumors is a simple and convenient method for assessment of RAS mutation. Different studies, including the PERSEIDA (6) and the AGEO RASANC study (7), have shown excellent concordance between the RAS mutational status found in tissue samples of CRC and those in liquid biopsies (6-8). Therefore, it is an excellent tool for assessing molecular changes in patients with mCRC. Serial liquid biopsies performed in our cohort of patients with mCRC treated with anti-angiogenics showed changes in the $R A S$ oncogene genotype in ctDNA. These results support the fact that $\mathrm{mCRC}$ is a dynamic condition with an evolving molecular profile over the course of the disease.

Selection of resistant clones following systemic therapy has been reported in a wide variety of tumors (9-11).

These surviving cells have a higher phenotypic plasticity and ability to grow and disseminate to distant organs (12). In our study, we observed a negative selection of $R A S$ mutant clones from plasma samples of patients treated with anti-angiogenic-based chemotherapy treatment, supporting previous data (13-15). Our results suggest that anti-VEGF treatment might play a critical role in this clonal selection.

However, our study was not able to investigate whether these changes were secondary to anti-VEGF treatment, chemotherapy, both, or just related to intrinsic genotypic alterations.

Our results support other data in the literature. Bouchahda et al. genotyped plasma of 16 primary $R A S$-mutated tumors from previously treated patients with $\mathrm{mCRC}$. Nine patients showed wild-type $R A S$ in their ctDNA. They took a step forward and offered patients with wild-type RAS anti-EGFR treatment (13). Likewise, Raimondi et al. reported the disappearance of $R A S$-mutant clones from the plasma of four patients initially carrying $R A S$-mutated primary tumors, all of which had been treated with anti-VEGF (14). These patients were also treated with anti-EGFR and some patients achieved a partial response. However, so far, the efficacy of anti-EGFR therapy in cases with wild-type $R A S$ determined in ctDNA with initially mutated tumors is unknown, and anti-EGFR treatment cannot be widely recommended for these patients until prospective randomized studies are held. Our results suggest that this area of research should be more profoundly explored and randomized studies should be performed.

The fact that 17 out of 23 of our patients experienced a selection of clones with wild-type $R A S$ in our study highlights the importance of performing molecular analysis prior to the administration of further lines of therapies. Moreover, considering that three of them reverted to their previous mutated status, our study supports the fact that clonal selection seems to be a dynamic feature and performing sequential biopsies at different times during the disease course might add substantial information regarding clonal selection.

The present study has some limitations. Firstly, the small number of patients included makes it difficult to extrapolate the results and establish precise conclusions. Secondly, the probability of detecting false-negative mutated tumors is plausible when using the Idylla ${ }^{\mathrm{TM}}$ kit, as reported by Vivancos et al. They compared Idylla ${ }^{\mathrm{TM}}$ quantitative $c t K R A S$ mutation test to OncoBEAM digital PCR assay, showing a lack of concordance in tumors with a mutated allelic fraction (MAF) below 1\% (16). Unfortunately, we were unable to use OncoBEAM $^{\circledR}$ or other next-generation sequencing techniques to validate our results. However, Vidal et al. showed the presence of MAF seems to be conditioned not by the number of metastatic sites but by the site of metastasis (17). In their study, patients with liver involvement had higher $R A S$ ctDNA ( $R A S$ MAF) compared with those without liver metastases $(p=0.001)$. In contrast, MAF from patients having only peritoneal metastases or lung metastatic involvement was much lower. In our study 14 patients had synchronous liver metastasis at the time of diagnosis and inclusion in the study. Hence, the expected higher mutated allelic fraction related to these tumors ensures the sensitivity of the Idylla ${ }^{\mathrm{TM}}$ technique. Finally, concomitant therapies (anti-angiogenics are commonly administered along with other cytostatic agents) are a potential source of bias in which $R A S$ mutational changes induced might also be induced by prior chemotherapies, indistinguishable from those induced by anti-angiogenics.

Nonetheless, the results of our study support the concept that anti-angiogenic therapy might induce changes in $R A S$ mutational status in patients with mCRC. Liquid biopsy is a minimally invasive and easily tool with promising results for dynamic evaluation of $R A S$ mutations. It can be performed at different periods of the disease and might guide the selection of targeted treatments, such anti-EGFR therapy. Assessment of changes from $R A S$-mutated to wild-type $R A S$ variants in patients with metastatic CRC and evaluation of anti-EGFR therapy in this context warrants further investigation and prospective randomized studies are required. 


\section{Conflict of Interest}

On behalf of all Authors, the corresponding author states that there are no conflicts of interest.

\section{Authors' Contributions}

EC, ML-G and BGS: Designed the research. ML-G, BGS, PDD-L and CG-R: wrote the article. EC, AJG, CG-R and FZT: contributed in different ways to the writing. PDD-L, AMH and IL reviewed the article. FN and IJT-V developed the statistical analysis. BGS and JM-R performed the measurements. All Authors contributed to the article and approved the submitted version.

\section{Acknowledgements}

The Authors thank all the staff who contributed to the study, especially the nursing staff of the Day Hospital who facilitated the taking of the samples.

\section{References}

1 Van Cutsem E, Oliveira J and ESMO Guidelines Working Group: Advanced colorectal cancer: ESMO clinical recommendations for diagnosis, treatment and follow-up. Ann Oncol 20 Suppl 4: 6163, 2009. PMID: 19454465. DOI: 10.1093/annonc/mdp130

2 Gazzaniga P, Raimondi C, Nicolazzo C, Gradilone A and Cortesi E: ctDNA might expand therapeutic options for second line treatment of KRAS mutant mCRC. Annals of Oncology 28: v586, 2020. DOI: 10.1093/annonc/mdx390.044

3 Zeng M, Kikuchi H, Pino MS and Chung DC: Hypoxia activates the K-ras proto-oncogene to stimulate angiogenesis and inhibit apoptosis in colon cancer cells. PLoS One 5(6): e10966, 2010. PMID: 20532039. DOI: 10.1371/journal.pone.0010966

4 Yamada T, Matsuda A, Koizumi M, Shinji S, Takahashi G, Iwai T, Takeda K, Ueda K, Yokoyama Y, Hara K, Hotta M, Matsumoto $\mathrm{S}$ and Yoshida H: Liquid biopsy for the management of patients with colorectal cancer. Digestion 99(1): 39-45, 2019. PMID: 30554222. DOI: 10.1159/000494411

5 Schwartz LH, Seymour L, Litière S, Ford R, Gwyther S, Mandrekar S, Shankar L, Bogaerts J, Chen A, Dancey J, Hayes W, Hodi FS, Hoekstra OS, Huang EP, Lin N, Liu Y, Therasse P, Wolchok JD and de Vries E: RECIST 1.1 - Standardisation and disease-specific adaptations: Perspectives from the RECIST Working Group. Eur J Cancer 62: 138-145, 2016. PMID: 27237360. DOI: 10.1016/j.ejca.2016.03.082

6 Valladares-Ayerbes M, Alfonso PG, Luengo JM, Caceres PP, Vieitez JM, Cruz-Hernandez JJ, Llanos M, Giron CG, Cirera L and Vila AL: Concordance in RAS mutation status between liquid and solid biopsies in subjects with RAS wild-type (wt) metastatic colorectal cancer $(\mathrm{mCRC})$ in first-line treatment in Spain: PERSEIDA study (NCT02792478). Journal of Clinical Oncology 36(15_suppl): e15602-e15602, 2019. DOI: 10.1200/ JCO.2018.36.15_suppl.e15602

7 Grasselli J, Elez E, Caratù G, Matito J, Santos C, Macarulla T, Vidal J, Garcia M, Viéitez JM, Paéz D, Falcó E, Lopez Lopez C, Aranda E, Jones F, Sikri V, Nuciforo P, Fasani R, Tabernero J, Montagut C, Azuara D, Dienstmann R, Salazar R and Vivancos A: Concordance of blood- and tumor-based detection of RAS mutations to guide anti-EGFR therapy in metastatic colorectal cancer. Ann Oncol 28(6): 1294-1301, 2017. PMID: 28368441. DOI: $10.1093 /$ annonc/mdx 112

8 Bachet JB, Bouché O, Taieb J, Dubreuil O, Garcia ML, Meurisse A, Normand C, Gornet JM, Artru P, Louafi S, Bonnetain F, ThirotBidault A, Baumgaertner I, Coriat R, Tougeron D, Lecomte T, Mary F, Aparicio T, Marthey L, Taly V, Blons H, Vernerey D and Laurent-Puig P: RAS mutation analysis in circulating tumor DNA from patients with metastatic colorectal cancer: the AGEO RASANC prospective multicenter study. Ann Oncol 29(5): 12111219, 2018. PMID: 29438522. DOI: 10.1093/annonc/mdy061

9 Klein CA: Selection and adaptation during metastatic cancer progression. Nature 501(7467): 365-372, 2013. PMID: 24048069. DOI: $10.1038 /$ nature 12628

10 Faltas BM, Prandi D, Tagawa ST, Molina AM, Nanus DM, Sternberg C, Rosenberg J, Mosquera JM, Robinson B, Elemento O, Sboner A, Beltran H, Demichelis F and Rubin MA: Clonal evolution of chemotherapy-resistant urothelial carcinoma. Nat Genet 48(12): 1490-1499, 2016. PMID: 27749842. DOI: 10.1038/ng.3692

11 Cayrefourcq L, Thomas F, Mazard T, Assenat E, Assou S and Alix-Panabières C: Selective treatment pressure in colon cancer drives the molecular profile of resistant circulating tumor cell clones. Mol Cancer 20(1): 30, 2021. PMID: 33557844. DOI: 10.1186/s12943-021-01326-6

12 McGranahan N and Swanton C: Clonal heterogeneity and tumor evolution: Past, present, and the future. Cell 168(4): 613-628, 2017. PMID: 28187284. DOI: 10.1016/j.cell.2017.01.018

13 Bouchahda M, Saffroy R, Karaboué A, Hamelin J, Innominato P, Saliba F, Lévi F, Bosselut N and Lemoine A: Undetectable $R A S$-Mutant Clones in Plasma: Possible Implication for AntiEGFR Therapy and Prognosis in Patients With RAS-Mutant Metastatic Colorectal Cancer. JCO Precis Oncol 4: PO.19.00400, 2020. PMID: 33015528. DOI: 10.1200/PO.19.00400

14 Raimondi C, Nicolazzo C, Belardinilli F, Loreni F, Gradilone A, Mahdavian Y, Gelibter A, Giannini G, Cortesi E and Gazzaniga P: Transient disappearance of RAS mutant clones in plasma: A counterintuitive clinical use of EGFR inhibitors in RAS mutant metastatic colorectal cancer. Cancers (Basel) 11(1): 42, 2019. PMID: 30621206. DOI: 10.3390/cancers 11010042

15 Gazzaniga P, Raimondi C, Urbano F and Cortesi E: EGFR Inhibitor as second-line therapy in a patient with mutant RAS metastatic colorectal cancer: Circulating tumor DNA to personalize treatment. JCO Precision Oncology (2): 1-6, 2020. DOI: 10.1200/PO.17.00277

16 Vivancos A, Aranda E, Benavides M, Élez E, Gómez-España MA, Toledano M, Alvarez M, Parrado MRC, García-Barberán V and Diaz-Rubio E: Comparison of the clinical sensitivity of the Idylla platform and the OncoBEAM RAS CRC assay for KRAS mutation detection in liquid biopsy samples. Sci Rep 9(1): 8976, 2019. PMID: 31222012. DOI: 10.1038/s41598-019-45616-y

17 Vidal J, Muinelo L, Dalmases A, Jones F, Edelstein D, Iglesias M, Orrillo M, Abalo A, Rodríguez C, Brozos E, Vidal Y, Candamio S, Vázquez F, Ruiz J, Guix M, Visa L, Sikri V, Albanell J, Bellosillo B, López R and Montagut C: Plasma ctDNA RAS mutation analysis for the diagnosis and treatment monitoring of metastatic colorectal cancer patients. Ann Oncol 28(6): 13251332, 2017. PMID: 28419195. DOI: 10.1093/annonc/mdx 125

Received May 8, 2021 Revised May 31, 2021 Accepted June 5, 2021 